\title{
PYROLYSIS OF A BINARY MIXTURE OF COMPLEX HYDROCARBONS: REACTION MODELING
}

\author{
PHILLIP E. SAVAGE \\ Department of Chemical Engineering, University of Michigan, Ann Arbor, MI 48109-2136, U.S.A.
}

(First received 31 March 1989; accepted in revised form $19 \mathrm{July} \mathrm{1989)}$

\begin{abstract}
A reaction model was developed for the pyrolysis of binary mixtures of compounds whose reactions can be apportioned into three or fewer parallel chains coupled by chain transfer. A representative application of this model to the pyrolysis of mixtures of the asphaltene model compounds n-pentadecylbenzene (PDB) and n-tridecylcyclohexane (TDC) illustrates its utility. The model, along with its associated rate constant estimates, quantitatively correlated the experimental temporal variations of the product yields from the individual pyrolyses of PDB and TDC. Model results for binary mixtures of PDB and TDC showed that the pyrolysis rates for both compounds were accelerated by the addition of the second compound. For instance, the pyrolysis of $\mathrm{PDB}$ at an initial concentration of $10^{-4} \mathrm{M}$ proceeded at a $36 \%$ higher rate in the presence of TDC at an equal concentration. The rate of TDC pyrolysis at [TDC] $=10^{-4} \mathrm{M}$, on the other hand, increased more than nine-fold upon the addition of PDB at a concentration of $10^{-4} \mathrm{M}$. Unlike the accelerated reaction rates, product selectivities were largely insensitive to the presence of the second compound. These results are consistent with and can be explained on the basis of the influence of concentration on the relative kinetics of bimolecular and unimolecular propagation, chain transfer, and termination steps. The model results also lead to the identification of quantitative criteria for determining when an added compound can act as a rate accelerator. Finally, this study permits speculation into the effects of interactions between alkylaromatic and alkylnaphthenic moieties in asphaltenes on the pyrolysis pathways, products, and kinetics.
\end{abstract}

\section{INTRODUCTION}

Previous pyrolyses of complex, high molecular weight hydrocarbons that mimic the thermally reactive moieties in materials such as coal and heavy oils have provided substantial insight into the thermal reactions of these complex feedstocks. As an example, the pyrolysis of $n$-pentadecylbenzene (PDB) and ntridecylcyclohexane (TDC), compounds that mimic the long-chain n-alkylaromatic and n-alkylnaphthenic moieties in petroleum asphaltenes (e.g., the heptane-insoluble fraction of heavy oils), permitted resolution of their thermal reaction pathways, kinetics, and mechanisms (Savage and Klein, 1987, 1988, 1989b). The model compound pyrolysis kinetics and pathways were then used in a mathematical model, which included a stochastic description of the structure of petroleum asphaltenes, to simulate asphaltene pyrolysis (Savage and Klein, 1989a). All of the model predictions were in qualitative accord with experimental data from asphaltene pyrolyses, and some predictions agreed quantitatively.

Although pyrolyses of individual model compounds provide valuable reaction engineering information that can be used successfully in modeling, as evidenced above, such experiments are limited in that they provide no information about the interactions that can occur during the pyrolysis of complex, multicomponent, and multifunctional reactants such as petroleum asphaltenes. The potential for interactions exists because an active center (e.g., free radical) derived from one portion of a particular molecule in a complex reactant can react with a different moiety in one of the other molecules. Consequently, reaction pathways that are important for the pyrolysis of a single model compound might not be equally important for the model compound-like moiety in a complex substrate. Conversely, pathways unobserved in single-component pyrolyses might become operative during the pyrolysis of complex materials.

Evidence that reactions between active centers derived from two different compounds or between an active center from one compound and a second compound can affect reaction rates, product selectivities, and reaction pathways has been provided by the few (Rebick, 1983) previous pyrolyses of binary mixtures of high molecular weight compounds. Zhou and Crynes (1986), for example, pyrolyzed ethylphenol in dodecane and found that ethylphenol inhibited the rate of dodecane cracking and that dodecane accelerated the rate of ethylphenol conversion. They postulated that the interactions between the ethylphenolderived and dodecane-derived radicals were responsible for the differences between the pure compound and mixture pyrolysis results. Allen and Gavalas (1984) reported that the rates of decomposition of methylene and ether bridges in coal model compounds were enhanced when the compounds were pyrolyzed in 1,2-dihydronaphthalene (dialin). They suggested that an aromatic displacement reaction with atomic hydrogen (formed from dialin decomposition) accelerated the bridge decomposition.

In spite of the foregoing demonstration that interactions can be important during pyrolysis, there has been very limited work on the pyrolysis of binary mixtures of complex high molecular weight hydrocarbons. This gap in the literature combines with our 
interest in determining the effects of potential interactions during asphaltene pyrolysis to motivate the present work. This paper reports on a reaction model that simulates the pyrolysis of the asphaltene model compounds n-pentadecylbenzene (PDB) and ntridecylcyclohexane (TDC), both individually and in binary mixtures.

\section{REACTION PATHWAYS}

Figure 1(a) displays the pathways previously deduced (Savage and Klein, 1987) for PDB pyrolysis. The reaction product spectrum comprises two pairs of major products (toluene plus 1-tetradecene and styrene plus n-tridecane) and numerous minor products. These three product lumps arise because there are only three types of aliphatic positions in PDB with unique hydrogen abstraction or $\beta$-scission kinetics. Abstraction of an $\alpha$ hydrogen leads to a resonance-stabilized, secondary benzylic radical whereas abstraction of all other hydrogen atoms leads to less stable secondary $\left(2^{\circ}\right)$ alkyl radicals. Thus the $\alpha$ position possesses unique kinetics in the hydrogen abstraction step. The unique $\beta$-scission step is decomposition of the $\gamma$-PDB radical because this reaction leads to a resonance-stabilized, primary benzyl radical. Decomposition of all other PDB radicals, on the other hand, produces primary $\left(1^{\circ}\right)$ alkyl radicals. All non- $\alpha$ and non- $\gamma$ positions in PDB share roughly equal reactivities for hydrogen abstraction and for $\beta$-scission, and therefore products derived from reactions at these positions collectively constitute the third category.

The pathways previously deduced for TDC pyrolysis (Savage and Klein, 1988) are displayed in Fig. 1(b). These are analogous to those for PDB in that the product slate can be conveniently lumped together into two pairs of major products (cyclohexane plus 1-tridecene and methylene cyclohexane plus dodecane) and numerous minor products. These three product lumps arise because, similar to the directing influence of the aromatic ring in PDB, the naphthenic ring in TDC affords preferential formation of tertiary $\left(3^{\circ}\right)$ radicals in hydrogen abstraction steps and $2^{\circ}$ radicals in $\beta$-scission steps. That is, hydrogen abstraction at the ring carbon bearing the aliphatic chain, which produces a $3^{\circ}$ TDC radical, is the fastest abstraction step because abstraction at all other positions yields less stable $2^{\circ}$ radicals. Additionally, $\beta$ scission of the $\beta$-TDC radical, which produces a secondary cyclohexyl radical, is the unique decomposition step because all other $\beta$-scission steps lead to $1^{\circ}$ alkyl radicals. All non- $\beta$ and non- $3^{\circ}$ positions in TDC share roughly equal reactivities for both hydrogen abstraction and $\beta$-scission, and these TDC radicals lead to the formation of the minor products.

\section{REACTION MODEL}

The foregoing discussion of the pyrolysis pathways for PDB and TDC indicates that the essential features of the pyrolysis of both long-chain n-alkylbenzenes and $\mathbf{n}$-alkylcyclohexanes can be modeled as three parallel chain reactions. Figure 2 provides a set of freeradical reaction steps consistent with the reaction pathways described above (Savage and Klein, 1987, $1988,1989 \mathrm{~b}$ ), and Table 1 provides the chemical identities of the species in Fig. 2. The reaction mechanism includes a single initiation step, which for either compound occurs by homolytic dissociation of the weakest $\mathrm{C}-\mathrm{C}$ bond in the molecule. All possible radical recombination steps are included as termination reactions. The three parallel chains featured in the center of Fig. 2 lead to the two sets of major product pairs, denoted as $\beta_{1} H$ plus $Q_{1}$ and $\beta_{2} H$ plus $Q_{2}$, and also to the minor products denoted collectively as $\beta_{3} H$ plus $Q_{3}$. Chain transfer, depicted by the reactions in the two peripheral columns of Fig. 2, can occur via hydrogen abstraction from the reactant, $R$, by both $\mu$ and $\beta$ radicals. In this paper, $\beta$ radicals are defined as those that propagate chain reactions exclusively via bimolecular steps (e.g., hydrogen abstraction), whereas $\mu$ radicals can participate in unimolecular propagation steps (e.g., $\beta$-scission).

To probe the influence of interactions during the pyrolysis of binary mixtures of PDB and TDC, the
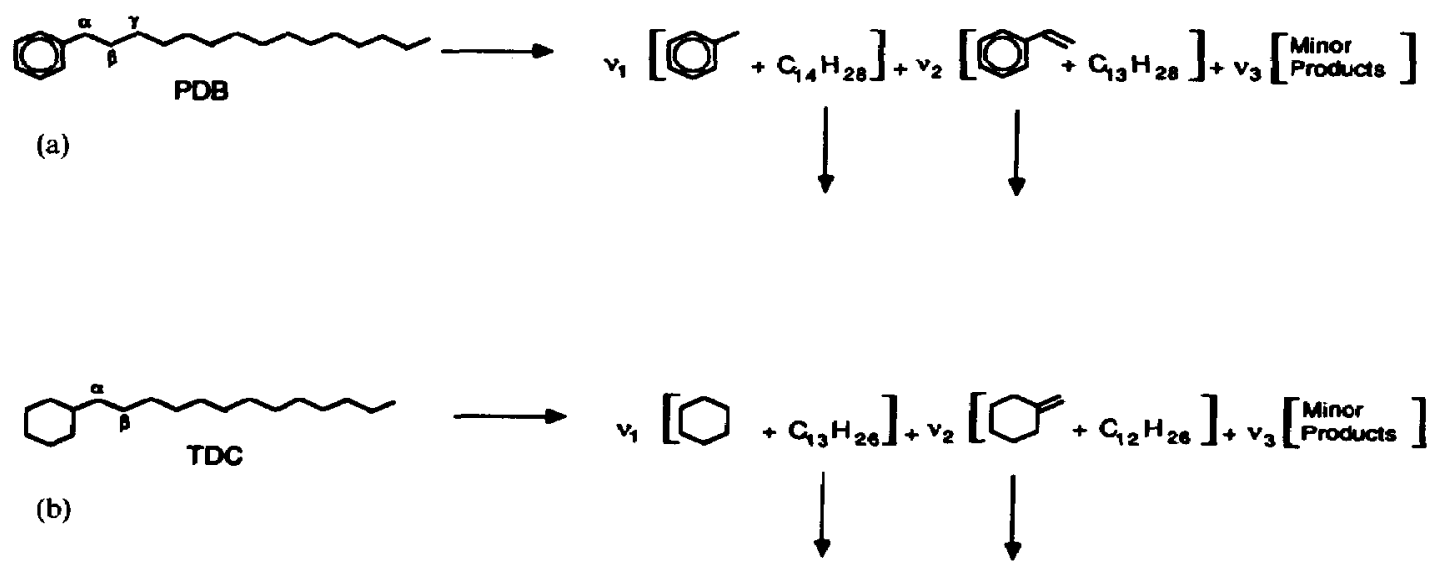

Fig. 1. Reaction pathways for model compound pyrolysis. (a) PDB, (b) TDC. 


$$
\begin{aligned}
& \text { Chain Tranofer } \\
& \beta_{1}+R \stackrel{k_{12}}{\longrightarrow} \beta_{1} H+\mu_{2} \\
& \mu_{1}+R \stackrel{k_{13}^{\prime}}{\longrightarrow} R+\mu_{2} \\
& \beta_{1}+R \stackrel{k_{12}}{\longrightarrow} \beta_{1} H+\mu_{1} \\
& \mu_{1} \stackrel{k_{\mu_{1}}}{\longrightarrow} \beta_{1}+Q_{1} \\
& \text { Chnin Transfer } \\
& \beta_{2}+R \stackrel{k_{21}}{\longrightarrow} \beta_{2} H+\mu_{1} \\
& \mu_{2}+R \stackrel{k_{21}^{\prime}}{\longrightarrow} R+\mu_{1} \\
& \beta_{2}+R \stackrel{k_{32}}{\longrightarrow} \beta_{2} H+\mu_{2} \\
& \mu_{2} \stackrel{k_{\mu_{2}}}{\longrightarrow} \beta_{2}+Q_{2} \\
& \beta_{2}+R \stackrel{k_{93}}{\longrightarrow} \beta_{2} H+\mu_{3} \\
& \mu_{2}+R \stackrel{\mu_{22}^{\prime}}{\longrightarrow} R+\mu_{3} \\
& \beta_{3}+R \stackrel{k_{33}}{\longrightarrow} \beta_{3} H+\mu_{3} \\
& \mu_{3} \stackrel{k_{m a}}{\rightarrow} \beta_{3}+Q s \\
& \beta_{3}+R \stackrel{\mu_{24}}{\longrightarrow} \beta_{3} H+\mu_{1} \\
& \mu_{3}+R \stackrel{k_{32}^{\prime}}{\longrightarrow} R+\mu_{1} \\
& \text { Termination } \\
& \boldsymbol{\beta}_{i}+\boldsymbol{\beta}_{j} \longrightarrow \text { Products } \\
& \mu_{i}+\beta_{i} \longrightarrow \text { Products } \\
& \mu_{i}+\mu_{j} \longrightarrow \text { Products } \\
& \beta_{3}+R \stackrel{k_{33}}{\longrightarrow} \beta_{3} H+\mu_{3} \\
& \mu_{3}+R \stackrel{k_{32}^{\prime}}{\longrightarrow} R+\mu_{2} \\
& \beta_{1}+R \stackrel{k_{13}}{\longrightarrow} \rho_{1} H+\mu_{3} \\
& \mu_{1}+R \stackrel{k_{j 3}^{\prime}}{\longrightarrow} R+\mu_{3}
\end{aligned}
$$$$
R \stackrel{k_{2}}{\longrightarrow} 2 \beta \quad \text { (Initiation) }
$$

Fig. 2. Free-radical reaction steps for pyrolysis of PDB and TDC individually.

Table 1. Chemical identities of species in Fig. 2 for PDB and TDC pyrolyses

$$
\boldsymbol{R}=\mathrm{PDB}
$$

\begin{tabular}{llll}
\hline$\beta_{1}$ & Benzyl radical & $\mu_{1}$ & $\gamma$-PDB radical \\
$\beta_{2}$ & Tridecyl radical & $\mu^{2}$ & $\alpha$-PDB radical \\
$\beta_{3}$ & Other non-PDB radicals & $\mu_{3}$ & Other PDB radicals \\
$\beta_{1} H$ & Toluene & $Q_{1}$ & 1 -Telradecene \\
$\beta_{2} H$ & Tridecane & $Q_{2}$ & Styrene \\
$\beta_{3} H$ & Minor products from PDB & $Q_{3}$ & Minor products from PDB
\end{tabular}

$R=\mathrm{TDC}$

\begin{tabular}{llll}
\hline$\beta_{1}$ & Cyclohexyl radical & $\mu_{1}$ & $\beta$-TDC radical \\
$\beta_{2}$ & Dodecyl radical & $\mu_{2}$ & 3 -TDC radical \\
$\beta_{3}$ & Other non-TDC radicals & $\mu_{3}$ & Other TDC radicals \\
$\beta_{1} H$ & Cyclohexane & $Q_{1}$ & 1-Tridecene \\
$\beta_{2} H$ & Dodecane & $Q_{2}$ & Methylene cyclohexane \\
$\beta_{3} H$ & Minor products from TDC & $Q_{3}$ & Minor products from TDC
\end{tabular}

mechanism given in Fig. 2 for the individual pyrolyses of these compounds was extended. This binary mixture pyrolysis model included the elementary steps in Fig. 2 for both PDB and TDC and also the addition of all likely interaction steps. The interaction steps take the form of hydrogen abstraction reactions in which a free-radical derived from one compound can abstract hydrogen from the second compound (and vice versa), and termination reactions, which involve the recombination of radicals derived from the two different substrates. Figure 3 displays the resulting mixture pyrolysis mechanism written in compact form. $R$ denotes a reactant molecule, and the superscripts $A$ and $B$ refer to $P D B$ and TDC, respectively. This model comprises 152 elementary steps; 40 describe the pyrolysis of PDB alone, 40 describe the pyrolysis of TDC alone, and the remaining 72 describe the interaction steps. Note that the mechanism of Fig. 3 depicts only the primary reaction pathways and thus will model only pyrolyses where primary reactions are emphasized.

\section{KINETICS DEVELOPMENT}

There are two different approaches for determining the pyrolysis kinetics for a compound via knowledge of the governing reaction mechanism. One approach combines the reaction rate laws implied by the elementary reaction steps in the mechanism with the relevant reactor design equation (e.g., for an isothermal, constant-volume batch reactor) to obtain a set of differential equations (typically stiff) that can be solved numerically. The number of differential equations is equal to the number of species in the mechanism, and solution of these equations provides the temporal variations of the concentrations of the different species. 
PDB PYRoLYsis

\begin{tabular}{|c|c|}
\hline $\begin{array}{l}\text { Initiation } \\
R^{A} \stackrel{k_{x}^{A}}{\longrightarrow} 2 \beta^{A}\end{array}$ & $\begin{array}{l}\text { Initiation } \\
R^{B} \stackrel{k=}{\longrightarrow} 2 Q^{B}\end{array}$ \\
\hline
\end{tabular}

Hydrogen Abetraction

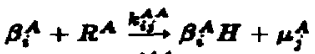
$\mu_{i}^{A}+R^{A} \stackrel{n_{i j}^{A}}{\rightarrow} R^{A}+\mu_{j}^{A}$

$\beta$-scigion
$\mu_{i}^{A} \stackrel{k \hat{A}_{i}}{\longrightarrow} \beta_{i}^{A}+Q_{i}^{A}$

Termination

$\beta_{i}^{A}+\beta_{j}^{A} \stackrel{k_{T}}{\longrightarrow}$ Products

$\mu_{i}^{A}+\beta_{j}^{A} \stackrel{k r}{\longrightarrow}$ Product.

$\mu_{\hat{i}}+\mu_{\hat{j}} \stackrel{\mu_{\boldsymbol{T}}}{\rightarrow}$ Product:
TDC PYROLYSIS

\section{INTERACTION STEPS}

Fig. 3. Reaction mechanism for pyrolysis of binary mixtures of $P D B$ and $I D C$.

Formulating and numerically integrating the large set of stiff differential equations governing hydrocarbon pyrolyses can be a tedious task, however, especially for complex reaction mechanisms such as the one considered here where there are 26 species (excluding termination products) and 152 reactions. Fortunately computer software exists that can free the user from this tedium, and Acuchem, one such software package developed by Braun et al. (1988), was used in the present work. Acuchem can accommodate reaction mechanisms comprising up to 99 species and 200 reactions, and to run the simulation the user needs only to provide the elementary steps in the mechanism and the corresponding rate constants. The program formulates and solves the governing differential equations and provides the temporal variations of the different species as output. These calculations were performed on an IBM PC-AT computer.

The second approach for determining reaction kinetics invokes the long-chain approximation (LCA) and the pseudo steady-state approximation (PSSA) and thereby provides a set of algebraic equations involving the concentrations of the free-radical species. Solution of these simultaneous equations for the pseudo-steady-state free-radical concentration admits calculation of the reaction rate and product selectivities.

These two approaches are complementary with respect to their ease of implementation and the information they provide. Direct integration of the stiff differential equations (i.e., the first approach) provides the temporal variations of the species' concentrations for pyrolyses in a constant-volume batch reactor, whereas solution of the algebraic equations (i.e., the second approach) provides the reactor-independent, pseudo-steady-state radical concentrations. The latter approach is quicker computationally, and one can more conveniently calculate reaction rates and product selectivities at a large number of different initial concentrations. Because the methods complement each other, both were employed in this work. In a later section the results from the two approaches will be compared to verify the validity of the LCA and PSSA for this complex system. The paragraphs that follow describe the kinetics development for the second approach in more detail. Note that this development, though applied here to mixtures of PDB and TDC, is completely general and can be applied to other compounds whose pyrolyses can be described by coupled parallel chains.

Expressions for the reaction rate and product selectivities were derived for the pyrolysis of binary mixtures of PDB and TDC on the basis of the mechanism in Fig. 3 by invoking the pseudo-steady-state and long-chain approximations (Gavalas, 1966). The PSSA permits approximation of the net rate of reaction for the free-radicals as being equal to zero, and the LCA permits us to neglect initiation and termination steps in the rate equations for the free-radical species involved in chain propagation. Applying these approximations to the rate law implied by the reactions in Fig. 3 for the $\beta_{i}$ radicals from compound $A$ (PDB) leads to eq. (1):

$r_{\beta_{i}^{A}}=0=k_{\mu_{i}}^{A} \mu_{i}^{A}-\sum_{j=1}^{3} k_{i j}^{A A} \beta_{i}^{A} R^{A}-\sum_{j=1}^{3} k_{i j}^{A B} \beta_{i}^{A} R^{B}$

which can then be solved for $\mu_{i}^{A}$ as

$$
\mu_{i}^{A}=y_{i}^{A} \beta_{i}^{A}
$$

where the dimensionless parameter $y_{i}^{A}$ is defined as

$$
y_{i}^{A}=\frac{k_{i}^{A A} R^{A}+k_{i}^{A B} R^{B}}{k_{\mu_{i}}^{A}}
$$

with $k_{i}^{A A}=\sum_{j=1}^{3} k_{i j}^{A A}$ and $k_{i}^{A B}=\sum_{j=1}^{3} k_{i j}^{A B}$. Similarly, the 
rate law for $\beta_{i}^{B}$ leads to

$$
\mu_{i}^{B}=y_{i}^{B} \beta_{i}^{B}
$$

where the expression for $y_{i}^{B}$ can be obtained from eq. (3) by transposing the $A$ and $B$ superscripts.

The double subscript notation employed for the hydrogen abstraction rate constants identifies, respectively, the attacking radical and the position attacked. The double superscript notation identifies, respectively, the substrate from which the attacking radical was derived and the substrate attacked. Primed and unprimed rate constants denote hydrogen abstraction from either of the substrates by $\mu$ and $\beta$ radicals, respectively. Thus $k_{i j}^{A B}$ is the rate constant for hydrogen abstraction at position $j$ in $R^{B}$ by $\beta_{i}^{A}$, and the resultant radical is $\mu_{j}^{\mathrm{B}}$.

The long-chain, steady-state rate expression for $\mu_{i}^{A}$ is given as eq. (5):

$$
\begin{aligned}
r_{\mu_{i}^{A}}=0= & \sum_{j=}^{3} k_{j i}^{A A} \beta_{j}^{A} R^{A}-k_{\mu_{i}}^{A} \mu_{i}^{A} \\
& -\sum_{j=1 \neq i}^{3} k_{i j}^{A A} \mu_{i}^{A} R^{A}+\sum_{j=1 \neq i}^{3} k_{j i}^{\prime A A} \mu_{j}^{A} R^{A} \\
& -\sum_{j=1}^{3} k_{i j}^{A B} \mu_{i}^{A} R^{B}+\sum_{j=1}^{3} k_{j i}^{\prime B A} \mu_{j}^{B} R^{A} \\
& +\sum_{j=1}^{3} k_{j i}^{B A} \beta_{j}^{B} R^{A} .
\end{aligned}
$$

The rate expression for $\mu_{i}^{B}$ is completely analogous and can be obtained by transposing the $A$ and $B$ superscripts in eq. (5).

Substituting eqs (2) and (4) for the concentrations of the $\mu_{i}^{A}$ and $\mu_{i}^{B}$ radicals, respectively, into eq. (5) provides equations involving only the $\beta_{i}$ radical concentrations. Equation (6) displays the resulting simplified expression:

$$
\begin{aligned}
\sum_{j=1 \neq j}^{3} Z_{j i}^{A A} \beta_{j}^{A} & +\sum_{j=1}^{3} Z_{j i}^{B A} \beta_{j}^{B} \\
& =\beta_{i}^{A}\left(\sum_{j=1 \neq i}^{3} Z_{j j}^{A A}+\frac{R^{B}}{R^{A}}\left(\sum_{j=1}^{3} Z_{i j}^{A B}\right)\right)
\end{aligned}
$$

where

$$
\begin{aligned}
& Z_{i j}^{A A}=k_{i j}^{A A}+y_{i}^{A} k_{i j}^{A A} \\
& Z_{i j}^{B A}=k_{i j}^{B A}+y_{i}^{B} k_{i j}^{\prime B A} .
\end{aligned}
$$

Expressions for $Z_{i j}^{R B}$ and $Z_{i j}^{A B}$ can be obtained by interchanging the $A$ and $B$ superscripts in eqs (7) and (8), respectively.

Equation (6) represents a set of three equations (for the three different values of $i$ ), and an analogous set of three equations can be derived for substrate $B$. Only five of these six equations are independent, however; thus we need an additional equation to solve for the six $\beta$ radical concentrations (i.e., $\beta_{i}^{A}$ and $\beta_{i}^{B}$ for $i=1,2$, 3). A balance on the total radical population provides this sixth equation, which then permits solution for the individual radical concentrations and ultimately the reaction rate and product selectivities.
The free-radical concentration changes only as a result of initiation and termination steps, thus applying the PSSA to the net rate of reaction of all radicals results in equating the rates of initiation and termination. Furthermore, because rate constants for radical recombinations are typically $10^{9.5 \pm 1.0} 1 / \mathrm{mol}-\mathrm{s}$ (Benson, 1976; Kerr, 1973), all termination rate constants are expected to be approximately equal. Collision theory, however, indicates that rate constants for the combination of two dissimilar radicals, $k_{T}$, should be roughly twice the value of the rate constants, $k_{T}^{\prime}$, for the combination of two identical radicals (Kerr, 1973). In formulating the overall radical balance, then we took all termination rate constants involving identical radicals to be equal to $k_{r}^{\prime}$, and the rate constants for the cross-combination reactions were set equal to $k_{T}=2 k_{T}^{\prime}$. The foregoing approximations combine with the initiation and termination steps depicted in Fig. 3 to give eq. (9) as the pseudo-steadystate radical balance:

$k_{I}^{A} R^{A}+k_{I}^{B} R^{B}=k_{T}^{\prime}\left[\sum_{i=1}^{3}\left(\mu_{i}^{A}+\mu_{i}^{B}+\beta_{i}^{A}+\beta_{i}^{B}\right)\right]^{2}$.

Eliminating $\mu_{i}^{A}$ and $\mu_{i}^{B}$ from eq. (9) via eqs (2) and (4), respectively, gives eq. (10):

$$
\begin{aligned}
{\left[\frac{k_{I}^{A} R^{A}+k_{I}^{B} R^{B}}{k_{T}^{\prime}}\right]^{1 / 2}=} & \sum_{i=1}^{3}\left[\beta_{i}^{A}\left(1+y_{i}^{A}\right)\right. \\
& \left.+\beta_{i}^{B}\left(1+y_{i}^{B}\right)\right] .
\end{aligned}
$$

This equation, eq. (6), and the analogous expressions for substrate $B$ provided the six simultaneous linear equations that were solved numerically for the $\beta$ radical concentrations. The initial reaction rates for PDB and TDC were then determined from the longchain rate expressions below:

$$
\begin{aligned}
r_{A}= & R^{A} \sum_{i=1}^{3}\left[\beta_{i}^{A}\left(k_{i}^{A A}-\frac{R^{B}}{R^{A}} k_{i}^{\prime A B} y_{i}^{A}\right)\right. \\
& \left.+\beta_{i}^{B}\left(k_{i}^{B A}+k_{i}^{\prime B A} y_{i}^{B}\right)\right] \\
r_{B}= & R^{B} \sum_{i=1}^{3}\left[\beta_{i}^{B}\left(k_{i}^{B B}-\frac{R^{A}}{R^{B}} k_{i}^{B A} y_{i}^{B}\right)\right. \\
& \left.+\beta_{i}^{A}\left(k_{i}^{A B}+k_{i}^{\prime A B} y_{i}^{A}\right)\right] .
\end{aligned}
$$

Relative rates for the pyrolysis of PDB (or TDC) were calculated as the ratio of the initial rate at a specified value of $R^{B} / R^{A}$ (or $R^{A} / R^{B}$ ) to the initial rate calculated for the pyrolysis of the pure compound. Equation (13) provides the expression for the relative rate of $\mathrm{PDB}$ pyrolysis, and an analogous equation can be derived for TDC pyrolysis:

Relative rate for $R^{A}=$

$\frac{\sum_{i=1}^{3}\left[\beta_{i}^{A}\left(k_{i}^{A A}-\left(R^{B} / R^{A}\right) k_{i}^{\prime A B} y_{i}^{A}\right)+\beta_{i}^{B}\left(k_{i}^{B A}+k_{i}^{\prime A A} y_{i}^{B}\right)\right]}{\sum_{i=1}^{3}\left[\beta_{i}^{A} k_{i}^{A A}\right]}$.

This relative rate, or ratio of initial rates, proved to be 
convenient for exploring the influence of the addition of a second compound on the pyrolysis kinetics of the first compound.

Finally, product selectivities were calculated as the rate of forming a particular product divided by the rate of reactant disappearance. For instance, the selectivity, $S$, of PDB $\left(R^{A}\right)$ to toluene $\left(\beta_{1}^{A} H\right)$ is given by

$$
\begin{aligned}
& S_{\beta_{1}^{A} H}=\frac{r_{\beta_{1}^{A} H}}{r_{A}} \\
& =\frac{\left(k_{1}^{A A}+\frac{R^{B}}{R^{A}} k_{1}^{A B}\right) \beta_{1}^{A}}{\sum_{i=1}^{3}\left[\beta_{i}^{A}\left(k_{i}^{A A}-\frac{R^{B}}{R^{A}} k_{i}^{A B} y_{i}^{A}\right)+\beta_{i}^{B}\left(k_{i}^{B A}+k_{i}^{\prime B A} y_{i}^{B}\right)\right]}
\end{aligned}
$$

and other product selectivities were calculated similarly.

\section{RATE CONSTANT SELECTION}

In order to calculate relative reaction rates, product selectivities, and the temporal variations of the reaction products' yields using the methods described in the previous section, numerical values of the rate constants for each of the elementary steps in Fig. 3 are required. This section provides estimates of the values of these rate constants at $400^{\circ} \mathrm{C}$, the temperature at which pyrolysis was simulated. These estimates were determined from thermochemical kinetics considerations and from values reported in the literature for similar types of reactions. In some cases, however, values of individual rate constants were adjusted slightly to provide better agreement between the available experimental data for PDB and TDC pyrolysis and the results of the reaction model simulation for their pure component pyrolyses. Treating some rate constants as semi-adjustable parameters in the model is merely an acknowledgement of the uncertainty inherent in any rate constant estimate (Allara and Shaw, 1980).
This uncertainty in rate constant estimates is apparent in the literature, which at times provides conflicting values of rate constants for identical reactions. For instance, Arrhenius parameters of $\left[\log _{10} A\left(\mathrm{~s}^{-1}\right)\right.$, $\left.E^{*}(\mathrm{kcal} / \mathrm{mol})\right]=[16.7,83.0],[15.3,77.3],[17.4,82]$, $[16.4,82.1]$, and $[16.2,82.1]$ have been used by Dente and Ranzi (1983), Purnell (1980), Benson (1976), Edelson and Allara (1980), and Sundaram and Froment (1978), respectively, for the homolytic dissociation of n-butane into two ethyl radicals. The corresponding values of the first-order rate constant at $400^{\circ} \mathrm{C}$ calculated from these different Arrhenius parameters vary by a factor of 18 . Note, however, that although there exists uncertainty in estimated rate constants even for relatively simple reactions, we have a measure of confidence in the rate constants used in the present simulation. This is because they display the expected relative reactivity trends, and as will be shown in a subsequent section, they provide quantitative correlation of the experimental temporal variations of product yields for PDB and TDC pyrolysis. Note also that the sensitivity of reaction models to uncertainty in estimated rate constants has been treated in the literature (Edelson and Allara, 1980; Turanyi et al., 1989).

The balance of this section provides the details of the methods used to estimate the rate constants for the initiation and termination, $\beta$-scission, and hydrogen abstraction reactions, respectively. Tables $2-4$ summarize the results of these rate constant estimates.

\section{Initiation and termination reactions}

The initiation step for PDB is homolytic dissociation of the bond between the $\alpha$ and $\beta$ carbons in the aliphatic chain. This reaction, which breaks the weakest bond in the molecule, produces a resonance-stabilized benzyl radical and a 1-tetradecyl radical. Benson (1976) reports that most unimolecular decomposition reactions have frequency factors of about $10^{16 \pm 1} \mathrm{~s}^{-1}$, and previous estimates of the frequency factor for decomposition of n-alkylaromatics

Table 2. Initiation and termination rate constants at $400^{\circ} \mathrm{C}$

\begin{tabular}{ll}
\hline \multicolumn{1}{c}{ Reaction } & \multicolumn{1}{c}{$\begin{array}{c}\text { Rate constant } \\
\left(\mathrm{s}^{-1} \text { or } 1 / \mathrm{mol} \cdot \mathrm{s}\right)\end{array}$} \\
\hline PDB $\rightarrow$ benzyl radical + tetradecyl radical & $2.35 \times 10^{-7}$ \\
TDC $\rightarrow$ cyclohexyl radical + tridecyl radical & $7.40 \times 10^{-10}$ \\
$R \cdot+R^{\prime} \rightarrow$ termination products & $3.16 \times 10^{8}$ \\
\hline
\end{tabular}

Table 3. $\beta$-Scission rate constants at $400^{\circ} \mathrm{C}$

\begin{tabular}{lc}
\hline \multicolumn{1}{c}{ Rcaction } & $\begin{array}{c}\text { Rate constant } \\
\left(\mathrm{s}^{-1}\right)\end{array}$ \\
\hline$\gamma$-PDB $\rightarrow$ benzyl radical + tetradecene & $4.10 \times 10^{5}$ \\
$\alpha-\mathrm{PDB} \cdot \rightarrow$ styrene + tridecyl radical & $6.24 \times 10^{2}$ \\
$\beta-\mathrm{TDC} \cdot \rightarrow$ cyclohexyl radical + tridecene & $3.72 \times 10^{4}$ \\
All other PDB $\cdot$ and TDC $\rightarrow 1^{\circ}$ radical + olefin & $1.50 \times 10^{4}$ \\
\hline
\end{tabular}


Table 4. Hydrogen abstraction rate constants at $400^{\circ} \mathrm{C}$

\begin{tabular}{lc}
\hline \multicolumn{1}{c}{ Reaction } & $\begin{array}{c}\text { Rate constant } \\
(1 / \mathrm{mol} \cdot \mathrm{s})\end{array}$ \\
\hline $1^{\circ}$ Alkyl radical $+2^{\circ}$ aliphatic hydrogen & $1.96 \times 10^{4}$ \\
$1^{\circ}$ Alkyl radical $+3^{\circ}$ aliphatic hydrogen & $5.50 \times 10^{4}$ \\
$1^{\circ}$ Alkyl radical $+2^{\circ}$ benzylic hydrogen & $1.27 \times 10^{5}$ \\
$2^{\circ}$ Alkyl radical $+2^{\circ}$ aliphatic hydrogen & $4.75 \times 10^{3}$ \\
$2^{\circ}$ Alkyl radical $+3^{\circ}$ aliphatic hydrogen & $1.33 \times 10^{4}$ \\
$2^{\circ}$ Alkyl radical $+2^{\circ}$ benzylic hydrogen & $3.29 \times 10^{4}$ \\
$3^{\circ}$ Alkyl radical $+2^{\circ}$ aliphatic hydrogen & $3.00 \times 10^{3}$ \\
$3^{\circ}$ Alkyl radical $+2^{\circ}$ benzylic hydrogen & $1.97 \times 10^{4}$ \\
$1^{\circ}$ Benzylic radical $+2^{\circ}$ aliphatic hydrogen & $4.54 \times 10^{2}$ \\
$1^{\circ}$ Benzylic radical $+3^{\circ}$ aliphatic hydrogen & $1.27 \times 10^{3}$ \\
$1^{\circ}$ Benzylic radical $+2^{\circ}$ benzylic hydrogen & $2.27 \times 10^{3}$ \\
$2^{\circ}$ Benzylic radical $+2^{\circ}$ aliphatic hydrogen & $2.25 \times 10^{2}$ \\
$2^{\circ}$ Benzylic radical $+3^{\circ}$ aliphatic hydrogen & $6.30 \times 10^{2}$ \\
\hline
\end{tabular}

have typically fallen at the lower end of this range (Ebert et al., 1978; Benson, 1976; Robaugh et al., 1981). The activation energy for this initiation reaction is expected to be equal to the bond dissociation energy (BDE) of about 68-69 kcal/mol (Benson, 1976) because the reverse reaction is unactivated. Based on these considerations a frequency factor of $10^{15.4} \mathrm{~s}^{-1}$ and an activation energy of $68 \mathrm{kcal} / \mathrm{mol}$ were selected for this initiation reaction. The resulting value of the rate constant at $400^{\circ} \mathrm{C}$ is $2.35 \times 10^{-7} \mathrm{~s}^{-1}$.

The most rapid initiation step for TDC is homolytic dissociation of the weakest $\mathrm{C}-\mathrm{C}$ bond in the molecule: the bond between the cyclohexane ring and the $\alpha$ aliphatic carbon. Cleavage of this bond produces a secondary cyclohexyl radical and a $1^{\circ}$ n-tridecyl radical. Tsang (1972) provides Arrhenius parameters for the analogous reaction in ethylcyclohexane, and the resulting rate constant at $400^{\circ} \mathrm{C}$ is $1.5 \times$ $10^{-10} \mathrm{~s}^{-1}$. Allara and Shaw (1980) report Arrhenius parameters for the dissociation of 2-methylpentane into a $1^{\circ}$ and a $2^{\circ}$ alkyl radical, the same types of radicals produced in the initiation step for TDC, and the rate constant at $400^{\circ} \mathrm{C}$ determined from these Arrhenius parameters is $7.6 \times 10^{-10} \mathrm{~s} 1$. These literature valucs combinc to demonstrate that a rate constant of $7.4 \times 10^{-10} \mathrm{~s}^{-1}$, which was used in the reaction model, is a reasonable estimate for the initiation step in TDC.

Termination reactions, which are the reverse of initiation, typically proceed with a frequency factor of $10^{9.5 \pm 1.0} \mathrm{l} / \mathrm{mol}-\mathrm{s}$ and zero activation energy. The reaction model used a value of $10^{8.5} \mathrm{l} / \mathrm{mol}-\mathrm{s}$ for termination reactions involving unlike species and half this value for termination reactions involving identical species (Pryor, 1966).

Table 2 summarizes the initiation and termination rate constants used in the simulation of PDB and TDC pyrolysis at $400^{\circ} \mathrm{C}$.

\section{$\beta$-Scission reactions}

The reaction mechanism displayed in Fig. 3 includes only four unique types of $\beta$-scission reactions when these are categorized on the basis of the type of
$\mathrm{C}-\mathrm{C}$ bond being broken and the type of products formed. These four classes are as follows.

1. Decomposition of a $\gamma$-PDB radical to form a resonance-stabilized, primary benzyl radical and an olefin.

2. Decomposition of an $\alpha$-PDB radical to form styrene and a $1^{\circ}$ n-alkyl radical.

3. Decomposition of a $\beta$-TDC radical to form a $2^{\circ}$ cyclohexyl radical and an olefin.

4. Decomposition of all other PDB and TDC radicals to form a $1^{\circ}$-alkyl radical and an olefin. Note that either the radical or the olefin will contain the phenyl or cyclohexyl substituent.

The rate constant for the first $\beta$-scission reaction noted above was taken as $4.1 \times 10^{5} \mathrm{~s}^{-1}$ at $400^{\circ} \mathrm{C}$ based on the Arrhenius parameters of $\left[\log _{10} A\left(\mathrm{~s}^{-1}\right)\right.$, $\left.E^{*}(\mathrm{kcal} / \mathrm{mol})\right]=[14.8,28.3]$ reported by Poutsma and Dyer (1982) for the analogous reaction in diphenylbutane pyrolysis. Poutsma and Dyer (1982) also provide an estimate of $[15.2,38.2]$ for the Arrhenius parameters for the rate constant for the second type of $\beta$-scission step, which leads to styrene. The corresponding value of this rate constant at $400^{\circ} \mathrm{C}$ is $624 \mathrm{~s}^{-1}$. The fourth type of $\beta$-scission step is analogous to the decomposition of an aliphatic radical to produce a $1^{\circ}$ alkyl radical and an olefin. Edelson and Allara (1980), Kerr (1973), and Ranzi et al. (1983) provide estimates of the Arrhenius parameters for this type of reaction, and the corresponding values of the rate constants at $400^{\circ} \mathrm{C}$ are about $10^{4} \mathrm{~s}^{-1}$. We selected a value of $1.5 \times 10^{4} \mathrm{~s}^{-1}$ for use in the reaction model.

We found no previous estimates of the Arrhenius parameters for a $\beta$-scission reaction leading to formation of a secondary cyclohexyl radical, as in $\beta$-scission step 3 above. Edelson and Allara (1980), however, estimated the rate constant for $\beta$-scission of the 4 methyl-2-pentyl radical, which forms propylene and a secondary 2-propyl radical, to be $1.75 \times 10^{4} \mathrm{~s}^{-1}$ at $400^{\circ} \mathrm{C}$. Furthermore, Ranzi et al. (1983) suggest that, other things being equal, $\beta$-scission to a $2^{\circ}$ radical 
should be about four times as rapid as $\beta$-scission to a $1^{\circ}$ radical at $400^{\circ} \mathrm{C}$. Thus our estimate of $1.5 \times 10^{4} \mathrm{~s}^{-1}$ for $\beta$-scission to a $1^{\circ}$ radical suggests that the rate constant for $\beta$-scission to a $2^{\circ}$ radical should be about $6.0 \times 10^{4} \mathrm{~s}^{-1}$. The foregoing considerations provided initial estimates for this rate constant, and comparing the results of the simulations for TDC with experimental data for TDC pyrolysis (Savage and Klein, 1988) revealed that a value of $3.72 \times 10^{4} \mathrm{~s}^{-1}$ provided a good representation of the experimental observations. We used this value in the mixture pyrolysis model. Table 3 summarizes the $\beta$ scission rate constant values used in the reaction model.

\section{Hydrogen abstraction reactions}

Hydrogen abstraction reactions were grouped according to the type of abstracting radical and the type of hydrogen atom being abstracted for the purposes of estimating their rate constants. These two items should be the most important ones in determining the relative rate constants (Dente and Ranzi, 1983), and classifying the reactions in this way reduced the number of unique hydrogen abstraction rate constants that had to be estimated from 66 to 13 . The procedure used in estimating these hydrogen abstraction rate constants was to estimate the seven rate constants required for the PDB pyrolysis model first and verify these estimates by simulating PDB pyrolysis. Then the additional three rate constants required for TDC pyrolysis were estimated and verified by simulating $T D C$ pyrolysis. Finally, the remaining three rate constants that appear only in the mixture pyrolysis model were estimated. The following paragraphs provide additional details, and Table 4 summarizes the rate constant estimates for the hydrogen abstraction reactions at $400^{\circ} \mathrm{C}$. All rate constants are on a per hydrogen atom basis, thus they were multiplied by the number of abstractable hydrogen atoms before use in the pyrolysis simulations.

Poutsma and Dyer's (1982) work with diphenylbutane pyrolysis provides estimates for many of the rate constants required for the PDB simulation. Using the Arrhenius parameters they reported, one can calculate rate constants at $400^{\circ} \mathrm{C}$ for a $1^{\circ}$ alkyl radical abstracting a $2^{\circ}$ aliphatic hydrogen as $1.96 \times 10^{4} \mathrm{l}$ mol-s and for abstracting a secondary benzylic hydrogen as $1.27 \times 10^{5} 1 / \mathrm{mol}-\mathrm{s}$. Abstraction of a secondary benzylic hydrogen proceeded with a rate constant of $3.29 \times 10^{4} \mathrm{l} / \mathrm{mol}-\mathrm{s}$ when a $2^{\circ}$ alkyl radical was the attacking species and with a rate constant of $2.27 \times 10^{3} 1 / \mathrm{mol}-\mathrm{s}$ when a primary benzyl radical was attacking. This latter value is also in good accord with the Arrhenius parameters reported by Miller and Stein (1981) for the analogous reaction in 1,2diphenylethane pyrolysis. Poutsma and Dyer (1982) also estimated the Arrhenius parameters for abstraction of a $2^{\circ}$ aliphatic hydrogen by a primary benzyl radical, and this rate constant is $4541 / \mathrm{mol}-\mathrm{s}$ at $400^{\circ} \mathrm{C}$.
All of the values noted above were used without adjustment in the reaction model.

Poutsma and Dyer's (1982) estimate of the value of the rate constant for abstraction of a secondary aliphatic hydrogen by a secondary benzylic radical at $400^{\circ} \mathrm{C}$ is $118 \mathrm{l} / \mathrm{mol}-\mathrm{s}$, but an adjusted value of $225 \mathrm{l} /$ mol-s provided better correlation of the experimental results for $\mathrm{PDB}$ pyrolysis. The final abstraction rate constant needed for PDB pyrolysis was for abstraction of a $2^{\circ}$ aliphatic hydrogen by a $2^{\circ}$ alkyl radical. Allara and Shaw (1980) report Arrhenius parameters of $\left[\log _{10} A(1 / \mathrm{mol}-\mathrm{s}), E^{*}(\mathrm{kcal} / \mathrm{mol})\right]=[7.9,12.3]$ for abstraction of a $2^{\circ}$ hydrogen in n-propane by a 2 butyl radical, and these parameters lead to $8.05 \times 10^{3}$ $1 / \mathrm{mol}-\mathrm{s}$ as the rate constant at $400^{\circ} \mathrm{C}$. This value was too high for PDB pyrolysis, thus an adjusted value of $4.75 \times 10^{3} \mathrm{l} / \mathrm{mol}-\mathrm{s}$, which provided better accord with experimental results, was used in the model.

To simulate the pyrolysis of TDC and subsequently the pyrolysis of PDB-TDC mixtures, six additional hydrogen abstraction rate constants were required, and these all involved reactions at the tertiary carbon in TDC. Unfortunately, the literature provides substantially fewer rate constants for hydrogen abstraction reactions involving tertiary positions than it does for primary and secondary positions. We can estimate these rate constants, however, by assuming that the frequency factor in the hydrogen abstraction rate constant depends only on the abstracting radical and that the activation energy depends only on the type of hydrogen being abstracted (Dente and Ranzi, 1983). These widely adopted simplifications allow us to use relative reactivities reported in the literature to estimate the required rate constants.

Kerr (1973), for example, provides the relative reactivity of ethyl radicals in abstracting different types of hydrogen atoms at $182^{\circ} \mathrm{C}$. These data reveal that the ratio of abstraction rates at $3^{n}$ positions to $2^{n}$ positions was 4.0. If this difference in reactivity can be entirely attributed to differences in the activation energy then $\Delta E^{*}=1.2 \mathrm{kcal} / \mathrm{mol}$, and the value of the rate constant ratio at $400^{\circ} \mathrm{C}$ would be 2.5 . Similarly, data reported by $\mathrm{K}$ issin (1987) for hydrogen abstraction reactions at $250^{\circ} \mathrm{C}$ lead to a $\Delta E^{*}$ for abstraction at $3^{\circ} \mathrm{vs} 2^{\circ}$ positions of $1.4 \mathrm{kcal} / \mathrm{mol}$. This value implies a ratio of rate constants of 2.9 at $400^{\circ} \mathrm{C}$. Note that this latter value of $\Delta E^{*}$ is in $\operatorname{good}$ accord with the estimated $3 \mathrm{kcal} / \mathrm{mol}$ difference in BDE between $3^{\circ}$ and $2^{\circ} \mathrm{C}-\mathrm{H}$ bonds (Benson, 1976) and an Evans-Polanyi $\alpha$ of 0.5 , which is frequently used to correlate the kinetics of hydrogen abstraction from alkanes (Russell, 1973). The foregoing considerations indicate that a value of the rate constant ratio between 2.5 and 2.9 would be consistent with the literature. Using a value of 2.8 in the pyrolysis model provided hydrogen abstraction rate constants for $3^{\circ}$ positions that permitted accurate correlation of the available experimental data for TDC pyrolysis. The resulting values of the rate constants for abstraction of $3^{\circ}$ hydrogen atoms were then $5.5 \times 10^{4}$ for abstraction by a $1^{\circ}$ aliphatic radical, $1.33 \times 10^{4}$ for abstraction by a $2^{\circ}$ 
aliphatic radical, $1.27 \times 10^{3}$ for abstraction by a primary benzyl radical, and $6.30 \times 10^{2} \mathrm{l} / \mathrm{mol}-\mathrm{s}$ for abstraction by a secondary benzylic radical.

The two rate constants remaining to be estimated were for hydrogen abstraction by a $3^{\circ}$ radical. There are few data in the literature for such abstraction reactions, thus accurate estimation of these rate constants is difficult. One can determine order-of-magnitude estimates, however, by considering the relative stabilities of the abstracting radicals. For instance, a $3^{\circ}$ radical should be more reactive in hydrogen abstraction steps than a primary benzyl radical, which is more stable, but less reactive than a $2^{\circ}$ alkyl radical, which is less stable. Thus the rate constant for abstraction of a $2^{\circ}$ aliphatic hydrogen by a $3^{\circ}$ radical should be bounded by $4.54 \times 10^{2}$ and $4.75 \times 10^{3} \mathrm{l} / \mathrm{mol}-\mathrm{s}$, the earlier estimates for abstraction of this hydrogen by primary benzylic and $2^{\circ}$ radicals, respectively. Several values within this range were examined, and $3.0 \times 10^{3} \mathrm{l} / \mathrm{mol}-\mathrm{s}$ provided the best correlation of experimental results for TDC pyrolysis. Using the same radical stability arguments and the earlier rate constant estimates, one expects the rate constant for abstraction of a secondary benzylic hydrogen by a $3^{\circ}$ radical to be bounded by $2.27 \times 10^{3}$ and $3.29 \times 10^{4} 1 /$ mol-s. A value of $1.97 \times 10^{4} \mathrm{l} / \mathrm{mol}-\mathrm{s}$ was used in the reaction model for this rate constant.

\section{MODEL RESULTS}

This section presents the results of pyrolysis simulations for PDB and TDC individually and in binary mixtures. Results from the former simulations will be compared to and shown to be consistent with experimental data taken from the literature. No data have been reported for the mixture pyrolyses and thus no comparisons are possible here.

\section{Pure component pyrolyses}

To verify that the reaction mechanism displayed in Fig. 2 combined with the rate constants in Tables $2-4$ to provide an accurate description of the individual neat pyrolyses of PDB and TDC, Acuchem simulated these pyrolyses at $400^{\circ} \mathrm{C}$, and the simulation results were then compared with experimental data. More specifically, the calculated temporal variations of the molar yields of the reactant and of two of its major pyrolysis products were compared with their experimentally determined temporal variations.

Figure 4 displays these comparisons for $\mathrm{PDB}$ pyrolysis at $400^{\circ} \mathrm{C}$. The agreement between the calculated molar yields, depicted as solid lines, and the experimental molar yields (Savage and Klein, 1987), presented as symbols, is very good for both toluene and n-tridecane as evidenced by inspection of Fig. 4(a). Note that the yields of the other two major products from PDB pyrolysis (i.e., 1-tetradecene and styrene), were not included in this comparison because, whereas the reaction model includes only primary reactions, these unsaturated products underwent very rapid secondary reactions. Figure $4($ b) shows that the selected
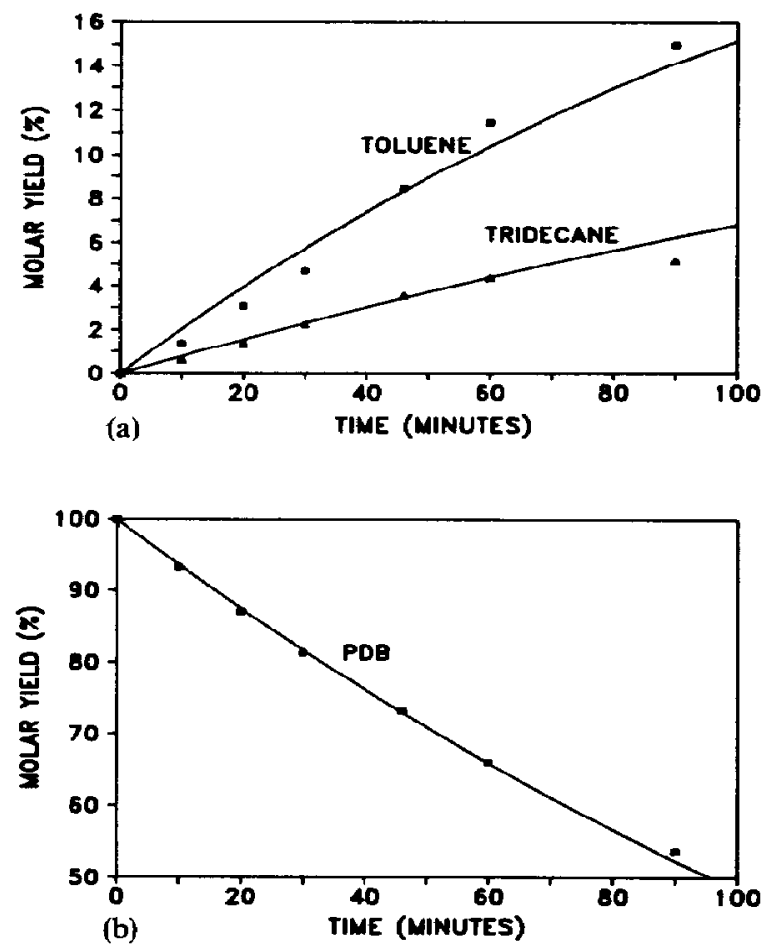

Fig. 4. PDB pyrolysis at $400^{\circ} \mathrm{C}$ : comparison of experiments and Acuchem simulation. (a) Toluene and n-tridecane molar yields, (b) PDB molar yield.

rate constants also provided accurate correlation of the kinetics of PDB disappearance. Here the data points represent the $\mathrm{PDB}$ yields calculated from the first-order rate constant reported by Savage and Klein (1987).

Figure 5 provides similar comparisons between experimental and model results for TDC pyrolysis at $400^{\circ} \mathrm{C}$. The primary pyrolysis products from TDC that underwent rapid secondary decomposition reactions (e.g., methylene cyclohexane and 1-tridecene) were excluded from this comparison. Inspection of Fig. 5(a) reveals excellent accord between the Acuchem calculations and the experimentally determined (Savage and Klein, 1988) molar yields for cyclohexane and n-dodecane. Figure 5(b) shows that the reaction model and the selected parameter values accurately represented the observed TDC pyrolysis kinetics. Once again, the data points in Fig. 5(b) represent molar yields calculated from the rate constant reported by Savage and Klein (1988) for TDC pyrolysis at $400^{\circ} \mathrm{C}$.

The quantitative agreement between the results from experiments and the simulations for both PDB and TDC pyrolyses shows that the postulated reaction mechanism and the selected rate constants are consistent with experimental observations.

\section{Mixture pyrolysis}

Because the pyrolysis of binary mixtures of PDB and TDC was simulated using the kinetics develop- 

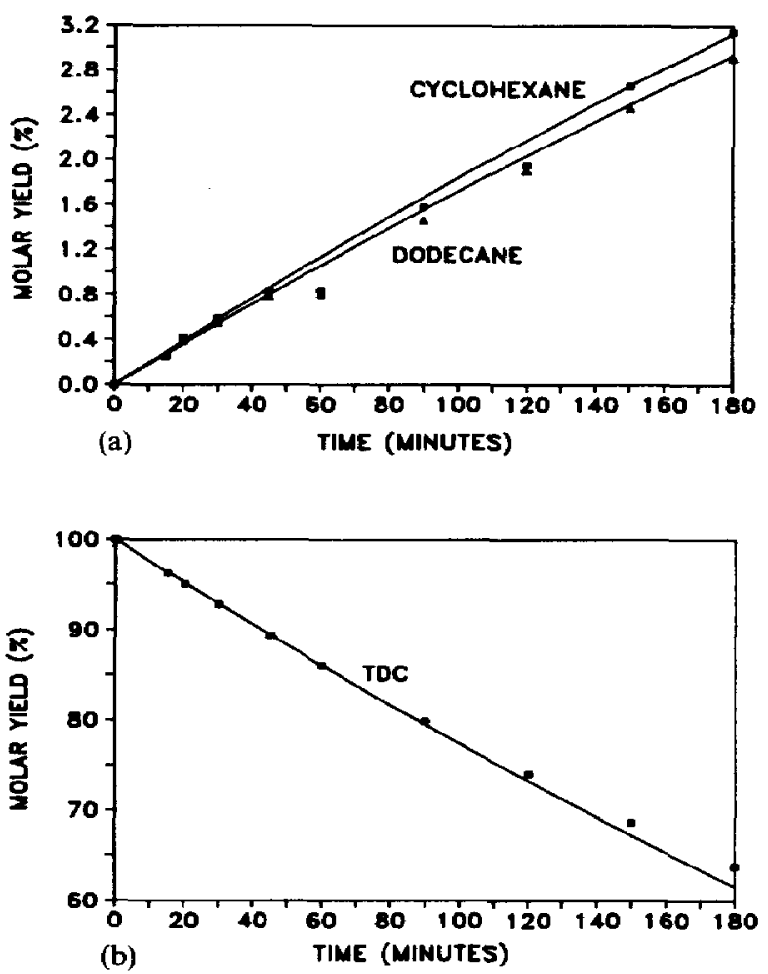

Fig. 5. TDC pyrolysis at $400^{\circ} \mathrm{C}$ : comparison of experiments and Acuchem simulation. (a) Cyclohexane and n-dodecane molar yields, (b) TDC molar yield.

ment that involved the pseudo-steady-state and longchain approximations, it is instructive to check the validity of these assumptions prior to presenting the model results. To verify the validity of the PSSA and the existence of long chains in the pyrolysis of binary mixtures of PDB and TDC, the reaction rates for both PDB and TDC and the $\mu$ and $\beta$ radical concentrations were calculated using both Acuchem and the method that employed the PSSA and LCA. Table 5, which compares the results, shows that the initial rates calculated by the two methods differed by less than $2 \%$. The radical concentrations calculated from the two methods were also roughly the same. The largest discrepancy was $2.6 \%$, and the average deviation was $1.0 \%$. This close agreement of reaction rates and radical concentrations establishes the validity of the PSSA and LCA in the kinetics analysis for the pyrolysis of binary mixtures of PDB and TDC.

Equation (13) was used to calculate the relative reaction rates for $P D B$ and TDC, and Fig. 6 summarizes the results of these rate calculations. Fgure 6(a) displays the relative rate of PDB pyrolysis as a function of the relative concentration of TDC (i.e., [TDC]/ [PDB]). The curves are parametric in the absolute concentration of PDB. The effect of TDC on the relative rate of $\mathrm{PDB}$ pyrolysis is to accelerate the reaction, but the amount of this acceleration is modest. It is most significant at low PDB concentrations and at high relative TDC concentrations (e.g., high values of the $[T D C] /[\mathrm{PDB}]$ ratio). For instance, when $[P D B]=10^{-4} \mathrm{M}$, the presence of TDC at $10^{-4} \mathrm{M}$ results in a reaction rate $36 \%$ higher than the rate calculated for PDB in the absence of TDC. When $[\mathrm{PDB}]=1.0 \mathrm{M}$, however, the addition of an equal amount of TDC provides only a $1 \%$ increase in the relative reaction rate.

Figure $6(\mathrm{~b})$ shows the pronounced effect of added PDB on the relative rate of TDC pyrolysis, which increases more than nine-fold when $[$ TDC] $=$ $10^{-4} \mathrm{M}$ and $\mathrm{PDB}$ is present in an equal concentration. The influence of added PDB on TDC pyrolysis kinetics is greatest when the absolute TDC concentration is low and when the relative PDB concentration is high. The curves for [TDC] $=0.01$ and $1.0 \mathrm{M}$ both exhibit maxima in their relative rates, and these maxima occur at higher values of $[\mathrm{PDB}] /[\mathrm{TDC}]$ as [TDC] decreases.

Table 5. Comparison of reaction rates and radical concentrations: justification of the PSSA and LCA

\begin{tabular}{|c|c|c|c|c|}
\hline & \multicolumn{2}{|c|}{$[\mathrm{PDB}]=[\mathrm{TDC}]=1.0 \mathrm{~mol} / 1$} & \multicolumn{2}{|c|}{$[\mathrm{PDB}]=[\mathrm{TDC}]=0.0001 \mathrm{~mol} / 1$} \\
\hline & $\begin{array}{c}\text { Acuchem } \\
(t=120 \mathrm{~s})\end{array}$ & PSSA and LCA & $\begin{array}{c}\text { Acuchem } \\
(t=120 \mathrm{~s})\end{array}$ & PSSA and LCA \\
\hline $\begin{array}{l}r_{\mathrm{PDB}}(\mathrm{mol} / 1-\mathrm{s}) \\
r_{\mathrm{TPC}}(\mathrm{mol} / \mathrm{l}-\mathrm{s}) \\
\beta_{1}^{A}(\mathrm{~mol} / 1) \\
\beta_{2}^{A}(\mathrm{~mol} / 1) \\
\beta_{3}^{A}(\mathrm{~mol} / 1) \\
\beta_{1}^{B}(\mathrm{~mol} / 1) \\
\beta_{2}^{B}(\mathrm{~mol} / 1) \\
\beta_{3}^{B}(\mathrm{~mol} / 1) \\
\mu_{1}^{A}(\mathrm{~mol} / 1) \\
\mu_{2}^{A}(\mathrm{~mol} / 1) \\
\mu_{3}^{A}(\mathrm{~mol} / 1) \\
\mu_{1}^{B}(\mathrm{~mol} / 1) \\
\mu_{2}^{B}(\mathrm{~mol} / 1) \\
\mu_{3}^{B}(\mathrm{~mol} / 1)\end{array}$ & $\begin{array}{l}1.47 \times 10^{-4} \\
9.10 \times 10^{-5} \\
1.34 \times 10^{-9} \\
1.38 \times 10^{-11} \\
5.95 \times 10^{-11} \\
2.19 \times 10^{-11} \\
5.03 \times 10^{-12} \\
5.53 \times 10^{-11} \\
1.00 \times 10^{-10} \\
3.07 \times 10^{-8} \\
2.74 \times 10^{-9} \\
2.00 \times 10^{-10} \\
4.65 \times 10^{-10} \\
2.56 \times 10^{-9}\end{array}$ & $\begin{array}{l}1.44 \times 10^{-4} \\
9.13 \times 10^{-5} \\
1.35 \times 10^{-9} \\
1.38 \times 10^{-11} \\
5.89 \times 10^{-11} \\
2.19 \times 10^{-11} \\
5.01 \times 10^{-12} \\
5.50 \times 10^{-11} \\
1.02 \times 10^{-10} \\
3.09 \times 10^{-8} \\
2.75 \times 10^{-9} \\
2.00 \times 10^{-10} \\
4.68 \times 10^{-10} \\
2.57 \times 10^{-9}\end{array}$ & $\begin{array}{l}1.32 \times 10^{-8} \\
9.50 \times 10^{-9} \\
2.04 \times 10^{-10} \\
2.90 \times 10^{-11} \\
6.14 \times 10^{-11} \\
1.85 \times 10^{-11} \\
6.38 \times 10^{-12} \\
5.69 \times 10^{-11} \\
1.54 \times 10^{-15} \\
6.47 \times 10^{-12} \\
2.84 \times 10^{-13} \\
1.70 \times 10^{-14} \\
5.91 \times 10^{-14} \\
2.64 \times 10^{-13}\end{array}$ & $\begin{array}{l}1.34 \times 10^{-8} \\
9.58 \times 10^{-9} \\
2.04 \times 10^{-10} \\
2.95 \times 10^{-11} \\
6.17 \times 10^{-11} \\
1.86 \times 10^{-11} \\
6.46 \times 10^{-12} \\
5.75 \times 10^{-11} \\
1.58 \times 10^{-15} \\
6.61 \times 10^{-12} \\
2.88 \times 10^{-13} \\
1.74 \times 10^{-14} \\
6.03 \times 10^{-14} \\
2.69 \times 10^{-13}\end{array}$ \\
\hline
\end{tabular}




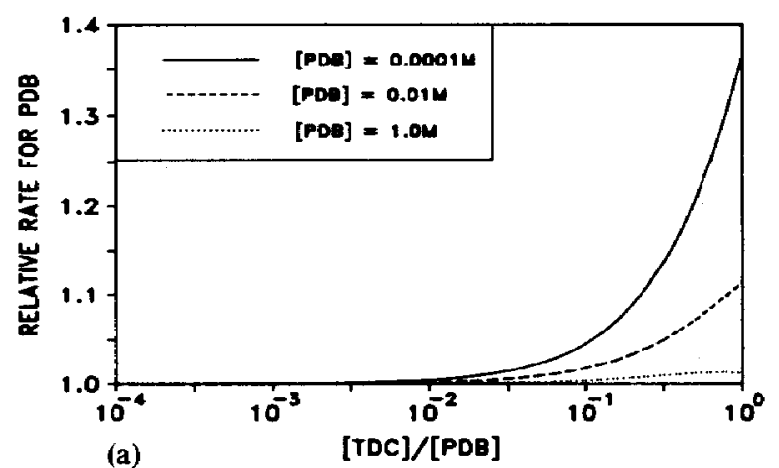

(a)

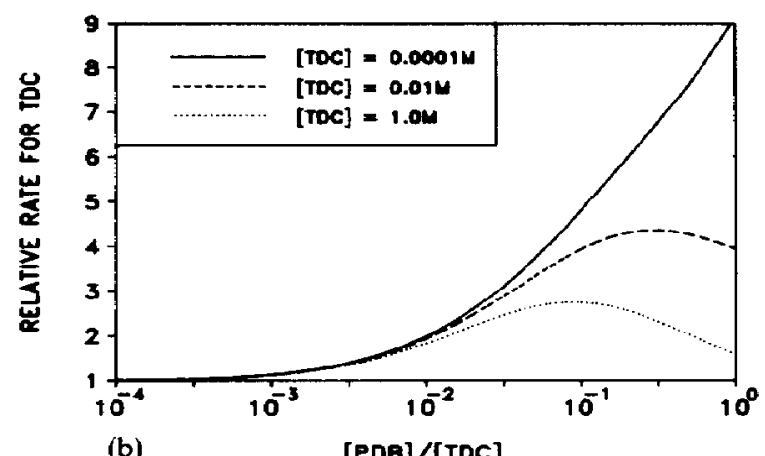

Fig. 6. Relative reaction rates for PDB-TDC copyrolysis. (a) $\mathrm{PDB}$ as major component in mixture, (b) TDC as major component in mixture.

Product selectivities, calculated from eq. (14) for toluene and analogous equations for the other products, are displayed in Figs 7 and 8. Figure 7, which shows the selectivity of PDB to toluene and to tridecane, reveals that the selectivities to both products are nearly insensitive to the addition of TDC except at high $[$ TDC $] /[\mathrm{PDB}]$ ratios and high $\mathrm{PDB}$ concentrations. Indeed, the largest change in selectivity is for toluene at $[\mathrm{PDB}]=1.0 \mathrm{M}$. For pure $\mathrm{PDB}$ the selectivity to toluene is 0.24 , and this value increases to 0.29 at a $[T D C] /[\mathrm{PDB}]$ ratio of unity. Further inspection of Fig. 7 reveals that the product selectivities are much stronger functions of the absolute PDB concentration than of the relative concentration of TDC. For instance, pyrolysis of pure PDB at $1.0 \mathrm{M}$ leads to toluene and tridecane selectivities of 0.24 and 0.15 , respectively, but these selectivities are 0.05 and 0.31 , respectively, at $[P D B]=10^{-4} \mathrm{M}$. This dramatic change in selectivity and shift in the major products from PDB pyroysis was noted previously and modeled by Savage and Klein (1989b) using the reaction mechanism of Fig. 2.

Figure 8 provides the selectivities of TDC to cyclohexane and dodecane as functions of the relative concentration of $\mathrm{PDB}$. The curves are parametric in the absolute TDC concentration. Once again, the effect of the addition of the second component (in this case PDB) is small. For instance, the selectivity to cyclohexane increases from 0.080 to only 0.082 when
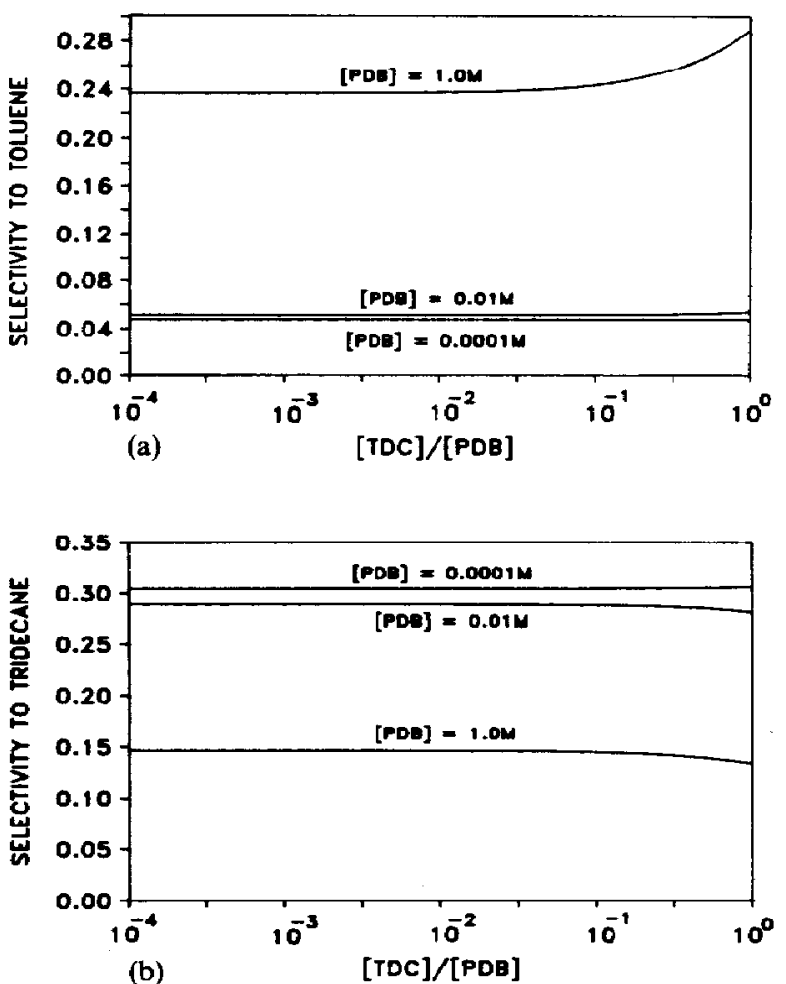

Fig. 7. Selectivity of PDB to major products for PDB-TDC copyrolysis. (a) Toluene, (b) tridecane.

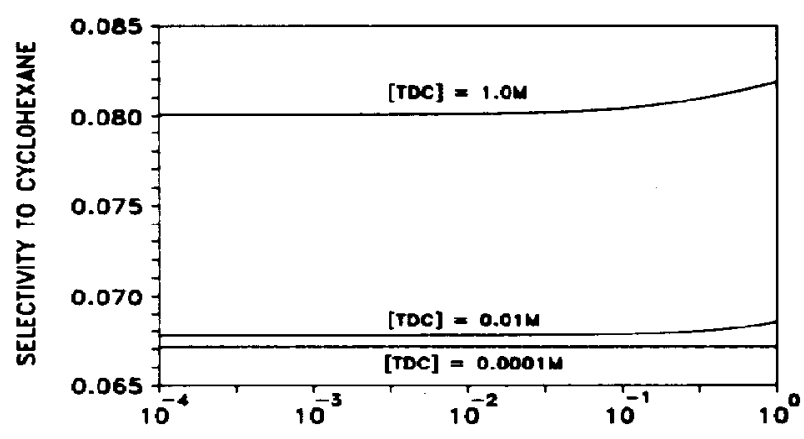

(a)

[PDE]/[TDC]

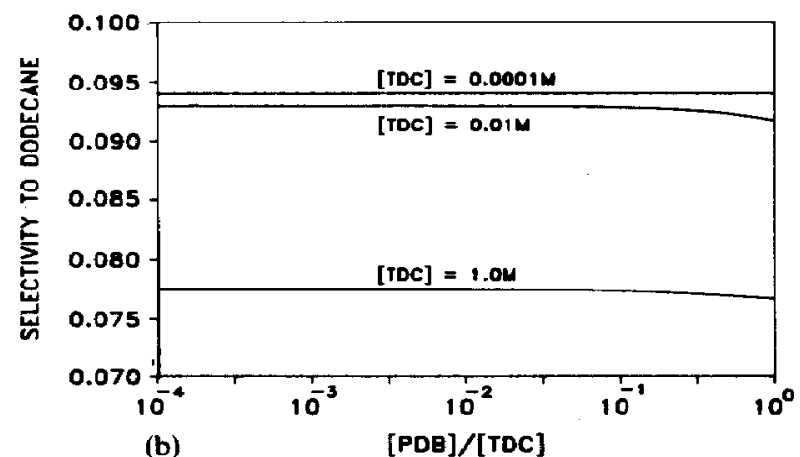

Fig. 8. Selectivity of TDC to major products for PDB-TDC copyrolysis. (a) Cyclohexane, (b) dodecane. 
$[\mathrm{TDC}]=1.0 \mathrm{M}$ and the relative $\mathrm{PDB}$ concentration increases from 0 to 1 .

\section{DISCUSSION OF MODEL RESULTS}

The results of the pyrolysis simulation for binary mixtures of PDB and TDC, presented in Figs 6-8 as relative reaction rates and product selectivities, exhibit four key trends as itemized below.

1. The rates of both PDB and TDC pyrolyses increases upon the addition of the other compound.

2. The amount of acceleration is highest at low absolute concentrations of the major component.

3. The relative rates of TDC pyrolysis exhibit maxima at high TDC concentrations.

4. The product selectivities demonstrate sensitivity to the presence of the second compound only at high absolute concentrations of the major component.

This section discusses these trends and shows that each is consistent with the expected influence of concentration on the relative kinetics of the different reaction steps for this complex system.

At low absolute substrate concentrations, bimolecular reactions in which the substrate participates compete at a disadvantage with unimolecular reactions, thus bimolecular hydrogen abstraction steps involved in chain propagation will be slow relative to unimolecular $\beta$-scission steps. Therefore, the concentration of $\beta$ radicals will be high and the concentration of $\mu$ radicals will, by comparison, be low. Table 5 , which shows that $\beta$ radicals are present in much higher concentrations than $\mu$ radicals when $[\mathrm{PDB}]=[\mathrm{TDC}]=0.0001 \mathrm{M}$, verifies this scenario. Consequently, in this low concentration region, the rate of the bimolecular hydrogen abstraction steps will control the overall pyrolysis rate. Thus, accelerating these abstraction reactions will also accelerate the apparent reaction rate. An added compound can accelerate the rate of hydrogen abstraction from the major component in the mixture if its addition increases the total radical concentration or if it facilitates replacement of one or more slow abstraction steps with faster reactions. As the following discussion will show, both of these modes of acceleration are important for the pyrolysis of binary mixtures of PDB and TDC, and the latter mode is responsible for the rate acceleration depicted in Fig. 6(a) for PDB pyrolysis.

The pyrolysis of PDB in the absence of TDC produces only $1^{\circ}$ aliphatic and $1^{\circ}$ benzyl radicals as the $\beta$ radical chain carriers. The benzyl radicals, being resonance stabilized, are relatively unreactive in hydrogen abstraction steps. When TDC is present, however, the TDC-derived $\mu$ radicals will undergo $\beta$ scission to produce $1^{\circ}$ and $2^{\circ}$ alkyl radicals, both of which are more reactive than. benzyl radicals. Thus, the effect of adding TDC is to increase the concentration of the more reactive alkyl radicals at the expense of the less reactive benzyl radicals. This shift in the distribution of $\beta$ radicals, which favors the production of more reactive chain carriers, accounts for the modestly accelerated rate of $\mathrm{PDB}$ pyrolysis upon the addition of TDC at low PDB concentrations.

The acceleration of TDC pyrolysis by the addition of PDB, as portrayed in Fig. 6(b), on the other hand, is due primarily to an increase in the total radical concentration. $\mathrm{PDB}$, which at $400^{\circ} \mathrm{C}$ undergoes homolytic dissociation (e.g., initiation) 317 times more rapidly than does TDC, acts as a free-radical initiator when added to TDC. Indeed, eq. (9) reveals that the total radical concentration for TDC pyrolysis increases by a factor of nearly 17 when $[\mathrm{PDB}] /[\mathrm{TDC}]$ increases from 0 to 1.0 . This substantially higher freeradical concentration leads to higher rates of hydrogen abstraction, and therefore higher TDC pyrolysis rates when the absolute concentration of TDC is low and hydrogen abstraction controls the apparent kinetics.

The second key feature of the model results was that the accelerating influence of the added compound decreases as the absolute concentration of the main component increases. This occurs because at higher concentrations, where bimolecular hydrogen abstraction reactions involving the substrate begin to compete more favorably with unimolecular $\beta$-scission steps, the net pyrolysis rate is no longer determined by the rate of abstraction. Therefore, the influence on the relative reaction of any shifts in the $\beta$ radical population favoring more reactive radicals or an increase in the total radical concentration is diminished.

The third trend noted at the beginning of this section was the appearance of maxima in the relative rate of TDC pyrolysis. These maxima occur only at moderate and high absolute TDC concentrations where $\mu$ radicals can be more abundant than $\beta$ radicals and unimolecular $\beta$-scission can be the controlling propagation step. Given these conditions, a maximum in the relative rate can appear because of the different influences that PDB has on the termination and propagation reactions for TDC. That is, when the amount of added PDB is small, its primary effect is to serve as a free-radical initiator and thus to increase the relative rate of TDC pyrolysis. At high relative PDB concentrations, however, the higher free-radical concentration that accompanies the addition of PDB increases the rate of the bimolecular chain termination reactions more than the rate of the controlling chain propagation steps (unimolecular $\beta$-scission). The net result of this effect is, therefore, to reduce the relative reaction rate at high relative concentrations and produce the maxima apparent in Fig. 6(b).

The final trend, slight shifts in product selectivities at high concentrations, is a result of chain transfer reactions by $\mu$ radicals. When such bimolecular hydrogen abstraction reactions are kinetically significant, they favor the formation of products from the 
$\mu$ radicals that possess the most rapid $\beta$-scission kinetics because these are the most likely to decompose rather than abstract hydrogen. Thus, conditions favoring chain transfer should enhance the production of toluene, which arises from the most rapid $\beta$-scission step in PDB pyrolysis (i.e., decomposition of the $\gamma$-PDB radical), and cyclohexane, which arises from the most rapid $\beta$-scission step in TDC pyrolysis (i.e., decomposition of the $\beta$-TDC radical). Inspection of the product selectivities at the high concentrations $(1.0 \mathrm{M})$ in Figs 7 and 8 confirms this hypothesis. These figures show an increase in the selectivity of PDB to toluene [Fig. 7(a)] and of TDC to cyclohexane [Fig. $8(a)]$ as the relative concentration of the added compound increases.

\section{IDENTIFICATION OF ACCELERATION CRITERIA}

The foregoing discussion of the simulation results leads to the identification of general criteria for determining conditions under which the addition of a second compound will accelerate the pyrolysis kinetics of a first compound. These criteria hold in concentration regions in which bimolecular hydrogen abstraction reactions are the controlling chain propagation steps (i.e., $\beta$ radicals are more abundant than $\mu$ radicals). Under such conditions, a second compound can act as a rate accelerator if:

1. It has an initiation rate constant much higher than that of the first compound;

2 . It produces $\beta$ radicals that are more reactive than those derived from the first compound.

These two observations can be developed into quantitative criteria for identifying the existence of these modes of acceleration in the pyrolysis of binary mixtures. The first observation, which requires inspection of the initiation rate constants for the two compounds, lead to the conclusion that the addition of compound $B$ to compound $A$ will accelerate the rate at which $A$ reacts if

$$
y_{i}^{A}<1 \text { and } \frac{k_{I}^{B}}{k_{I}^{A}}>1
$$

The first condition in this criterion is satisfied when $\beta_{i}^{A}$ radicals are more abundant than $\mu_{i}^{A}$ radicals [see eq. (2)], and thus, the rate of hydrogen abstraction determines the rate of chain propagation for $A$. The second condition is simply a statement of the wellknown influence of radical initiators on free-radical kinetics.

The second observation itemized above leads to another criterion. In this case, the addition of $B$ to $A$ can accelerate the rate at which $A$ reacts if

$$
y_{i}^{A}<1 \text { and } \frac{k_{i}^{B A}}{k_{i}^{A A}}>1 .
$$

The second condition in this criterion shows that the added compound $(B)$ can be an accelerator if the $\beta_{i}^{B}$ radicals derived from it are more reactive in hydro- gen abstraction steps than are the $\beta_{i}^{A}$ radicals derived from the original compound. Boudart (1987) has offered a similar criterion for acceleration in much simpler systems.

Recall that the foregoing criteria for acceleration are strictly valid only at low concentrations where hydrogen abstraction reactions control the rate of propagation (i.e., $y_{i}^{A}<1$ ). At higher concentrations, where unimolecular $\beta$-scission reactions can become the controlling propagation steps and where hydrogen abstraction by $\mu_{i}$ radicals can begin to play a role, the pyrolysis kinetics become considerably more complex. Indeed, the results of the mixture pyrolysis simulations showed that, in the high concentration regions, the accelerating influence of the second compound was diminished. In fact, preliminary work (Savage, 1988) with the present model but with different rate constant values showed that, under conditions where $\beta$-scission controlled the rate of chain propagation and where chain transfer by $\mu_{i}$ radicals occurred frequently, the addition of an alkylbenzene can inhibit, rather than accelerate, the pyrolysis rate for an alkylcyclohexane.

To demonstrate the utility of the criteria given above, we now apply them to the pyrolysis of mixtures of PDB (compound $A$ ) and TDC (compound $B$ ) for cases where the concentration of the major component is $10^{-4} \mathrm{M}$. Table 6 , which summarizes the results of the calculations, shows that both $y_{i}^{A}$ and $y_{i}^{B}$ are less than unity for all values of $i$. This being the case, $\beta$ radicals are the most abundant chain carriers, and we can therefore apply the two acceleration criteria given by eqs (15) and (16).

To apply the first criterion, we must calculate the ratio of initiation rate constants, $k_{I}^{A} / k_{I}^{B}$. The data in Table 2 show that this ratio is 317 . Therefore, the addition of a small amount of PDB to TDC should lead to a higher rate for the pyrolysis of TDC. Such acceleration is indeed observed in the mixture pyrolysis simulation results displayed in Fig. 6(b).

To apply the second criterion, we calculate the ratio of hydrogen abstraction rate constants, $k_{i}^{B A} / k_{i}^{A \mathcal{A}}$, and find that this ratio exceeds unity when $i=1$. Thus, the addition of TDC to PDB can lead to acceleration of PDB pyrolysis when $[P D B]=10^{-4} \mathrm{M}$. The effect of this behavior is displayed in Fig. 6(a).

implications to asphaltene pyrolysis

Asphaltenes, the heptane-insoluble fraction of heavy oils, are a complex mixture of the more polar,

Table 6. Evaluation of acceleration criteria: summary of calculations for $[\mathrm{PDB}]=[\mathrm{TDC}]=10^{-4} \mathrm{M}$

\begin{tabular}{ccccc}
\hline$i$ & $y_{i}^{A}$ & $k_{i}^{B A} / k_{i}^{A A}$ & $y_{i}^{B}$ & $k_{i}^{A B} / k_{i}^{B B}$ \\
\hline 1 & $7.6 \times 10^{-6}$ & 11.5 & $9.3 \times 10^{-4}$ & 0.09 \\
2 & $2.3 \times 10^{-1}$ & 1.00 & $9.4 \times 10^{-3}$ & 1.00 \\
3 & $4.7 \times 10^{-3}$ & 1.00 & $4.7 \times 10^{-3}$ & 1.00 \\
\hline
\end{tabular}


aromatic, and high molecular weight material in petroleum. It is generally agreed that asphaltenes contain aliphatic chains bonded to aromatic and naphthenic rings, and these moieties are important factors in determining the thermal reactivity of asphaltenes. Therefore, the results presented in the previous sections permit discussion of the potential effects of interactions on asphaltene pyrolysis pathways, products, and kinetics. To discern these effects, attention will be focused on the high concentration (e.g., 1.0 M) data of Figs 6-8 because these liquidphase conditions are more likely to reflect the conditions present during the neat pyrolysis of petroleum asphaltenes.

The data for $[P D B]=1.0 \mathrm{M}$ in Fig. 6(a) suggest that the presence of alkylnaphthenic moieties in asphaltene will have very little influence on the pyrolysis kinetics of the alkylaromatic moieties. The curve for $[T D C]=1.0 \mathrm{M}$ in Fig. 6(b), on the other hand, shows that the pyrolysis rate for long-chain alkylnaphthenic moieties in asphaltenes could be 2-3 times higher than the rate expected from the kinetics of TDC pyrolysis alone. Inspection of the $1.0 \mathrm{M}$ curves of Figs 7 and 8 , which show enhanced selectivities to toluene and cyclohexane at high relative concentrations, intimate slightly higher yields of methyl-substituted aromatics and unsubstituted naphthenic rings from asphaltene pyrolysis than would be indicated by the individual pyrolyses of PDB and TDC.

The foregoing extrapolation and application of the present results for the pyrolysis of binary mixtures of PDB and TDC to the pyrolysis of the analogous moieties in asphaltenes must be tempered by the realization that the two systems are different and that asphaltenes are considerably more complex (Speight, 1980; Bunger and Li, 1981). Moieties other than alkylaromatics and alkylnaphthenics exist in asphaltenes, and these could influence the pyrolysis kinetics. Thermally scissile thioether linkage, for instance, might function as free-radical initiators and accelerate the rate of asphaltene pyrolysis. Additionally, the importance of chain transfer by $\mu$ radicals, which leads to the higher toluene and cyclohexane selectivities in mixtures of PDB and TDC, could be diminished in asphaltene pyrolysis. This is because the asphaltene-derived $\mu$ radicals, which would contain a large polycyclic core rather than the single ring present in TDC and PDB, would be less mobile than their model compound counterparts. This reduced mobility would reduce the rate of the bimolecular chain transfer reactions and thereby reduce the influence of these reactions on product selectivities.

\section{SUMMARY AND CONCLUSIONS}

1. This paper provides a methodology for analyzing the kinetics of binary mixtures of compounds whose elementary steps can be apportioned into three or fewer parallel chain reactions. The methodology is general, and it can be applied to systems other than mixtures of PDB and TDC, the representative application presented here.
2. PDB accelerated TDC pyrolysis by acting as a free-radical initiator, and TDC accelerated PDB pyrolysis by producing aliphatic radicals at the expense of less reactive benzyl radicals. The amount of acceleration of the pyrolysis kinetics was a complex function of both the relative and absolute reactant concentrations.

3. Product selectivities in mixtures of PDB and TDC were relatively insensitive to the addition of the second compound except at high absolute concentrations where bimolecular chain transfer reactions involving $\mu$ radicals were important.

\section{NOTATION}

$A$ pre-exponential factor in Arrhenius equation $\left(\mathbf{s}^{-1}, 1 / \mathrm{mol} \cdot \mathbf{s}\right)$

$E^{*} \quad$ activation energy $(\mathrm{kcal} / \mathrm{mol})$

$k, k^{\prime} \quad$ rate constants $\left(\mathrm{s}^{-1}, 1 / \mathrm{mol} \cdot \mathrm{s}\right)$

$Q \quad$ reaction product in Figs 2 and 3

$R \quad$ substrate in Figs 2 and 3

$r \quad$ reaction rate $(\mathrm{mol} / 1 \cdot \mathrm{s})$

$S \quad$ selectivity

$t$ time (s)

$y$ dimensionless parameter defined in eq. (3)

$Z \quad$ dimensionless parameter defined in eqs (7) and (8)

\section{Greek letters}

$\alpha \quad$ first aliphatic position in alkyl substituent (adjacent to a ring)

$\beta$ radical reacting exclusively in bimolecular propagation steps

$\beta \quad$ second aliphatic position in alkyl substituent

$\beta H$ reaction product in Figs 2 and 3

$\gamma \quad$ third aliphatic position in alkyl substituent

$\mu$ radical reacting in unimolecular propagation steps

\section{Subscripts and superscripts \\ $A$ pertaining to PDB \\ $B$ pertaining to TDC \\ $i \quad$ summation index \\ I initiation \\ $j \quad$ summation index \\ $T$ termination \\ $\mu_{i} \quad$ pertaining to $\mu$ radicals or $\beta$-scission reactions \\ 1 pertaining to chain number 1 \\ 2 pertaining to chain number 2 \\ 3 pertaining to chain number 3}

\section{REFERENCES}

Allara, D. L. and Shaw, R., 1980, A compilation of kinetic parameters for the thermal degradation of n-alkane molecules. J. phys. Chem. Ref. Data 9, 523-559.

Allen, D. T. and Gavalas, G. R., 1984, Reactions of methylene and ether bridges. Fuel 63, 586-592.

Benson, S. W., 1976, Thermochemical Kinetics. John Wiley \& Sons, New York.

Boudart, M., 1987, Kinetics-assisted design of catalytic cycles. In Catalyst Design (Edited by Hegedus, L. L.), pp. 141-162. John Wilcy \& Sons, New York. 
Braun, W., Herron, J. T. and Kahaner, D. K., 1988, A computer program for modeling complex chemical reaction systems. Int. J. Chem. Kinetics 20, 51-62.

Bunger, J. W. and Li, N. C., 1981, Chemistry of Asphaltenes. ACS Adv. Chem. Ser. No. 195, American Chemical Society.

Dente, M. E. and Ranzi, E. M., 1983, Mathematical modeling of hydrocarbon pyrolysis reactions. In Pyrolysis: Theory and Industrial Practice (Edited by Albright, L. F., Crynes, B. L. and Corcoran, W. H.), pp. 133-175. Academic Press.

Ebert, K. H., Ederer, H. J. and Schmidt, P. S., 1978, Development of reaction models for complex gas phase reactions. In Chemical Reaction Engineering-Houston (Edited by Weekman, V. W. and Luss, D.) p. 313-324. ACS Symposium Series No. 65.

Edelson, D. and Allara, D. L., 1980, A computational analysis of the alkane pyrolysis mechsnism: sensitivity analysis of individual reaction steps. Int. J. Chem. Kinetics 12 , 605-621.

Gavalas, G. R., 1966, The long chain approximation in free radical reaction systems. Chem. Engng Sci. 21, 133-141.

Kerr, J. A., 1973, Rate processes in the gas phase. In Free Radicals (Edited by Kochi, J. K.), pp. 1-36. John Wiley \& Sons, New York.

Kissin, Y. V., 1987, Free-radical reactions of high molecular weight isoalkanes. Ind. Engng Chem. Res. 26, 1633-1638.

Miller, R. E. and Stein, S. E., 1981, Liquid-phase pyrolysis of 1,2-diphenylethane. $J$. phys. Chem. 85, 580-589.

Poutsma, M. L. and Dyer, C. W., 1982, Thermolysis of model compounds for coal. 3. Radical chain decomposition of 1,3-diphenylpropane and 1,4-diphenylbutane. $J$. org. Chem. 47, $4903-4914$.

Pryor, W. A., 1966, Free Radicals. McGraw-Hill, New York.

Purnell, J. H., 1980, Homogeneous alkane cracking: the route to quantitative description to very high conversion. In Frontiers of Free Radical Chemistry (Edited by Pryor, W. A.), pp. 93-115. Academic Press.

Ranzi, E., Dente, M., Pierucci, S. and Biardi, G., 1983, Initial product distributions from pyrolysis of normal and branched paraffins. Ind. Engng Chem. Fundam. 22, 132139.
Rebick, C., 1983, Pyrolysis of heavy hydrocarbons. In Pyrolysis: Theory and Industrial Practice (Edited by Albright, L. F., Crynes, B. L. and Corcoran W. H.), pp. 69-87. Academic Press.

Robaugh, D. A., Barton, B. D. and Stein, S. E., 1981, Thermal decomposition of propyl-, isobutyl-, and neopentylbenzene. J. phys. Chem. 85, 2378-2383.

Russell, G. A., 1973, Reactivity, selectivity, and polar effects in hydrogen atom transfer reactions. In Free Radicals (Edited by Kochi, J. K.), pp. 275-326. John Wiley \& Sons, New York.

Savage, P. E., 1988, Pyrolysis of a binary hydrocarbon mixture: reaction modeling. Prepr. Am. chem. Soc. Div. Fuel Chem. 33, 250-256.

Savage, P. E. and Klein, M. T., 1987, Asphaltene reaction pathways. 2. Pyrolysis of n-pentadecylbenzene. Ind. Engng Chem. Res. 26, 488-494.

Savage, P. E. and Klein, M. T., 1988, Asphaltene reaction pathways. 4. Pyrolysis of n-tridecylcyclohexane and 2ethyltetralin. Ind. Engng Chem. Res. 27, 1348-1356.

Savage, P. E. and Klein, M. T., 1989a, Asphaltene reaction pathways-V. Chemical and mathematical modeling. Chem. Engng Sci. 44, 393-404.

Savage, P. E. and Klein, M. T., 1989b, Kinetics of coupled reactions: lumping pentadecylbenzene pyrolysis into three parallel chains. Chem. Engng Sci. 44, 985-991.

Speight, J. G., 1980, The Chemistry and Technology of Petroleum. Marcel Dekker.

Sundaram, K. W. and Froment, G. F., 1978, Modeling of thermal cracking kinetics. 3. Radical mechanisms for the pyrolysis of simple paraffins, olefins, and their mixtures. Ind. Engng Chem. Fundam. 17, 174-182.

Tsang, W., 1972, Thermal decomposition of 3,4-dimethylhexane, 2,2,3-trimethylpentane, tertbutylcyclohexane, and related hydrocarbons. J. phys. Chem. 76, 143-156.

Turanyi, T., Berces, T. and Vajda, S., 1989, Reaction rate analysis of complex kinetic systems. Int. J. Chem. Kinetics 21, 83-99.

Zhou, P. and Crynes, B. L., 1986, Thermolytic reactions of o-ethylphenol. Ind. Engng Chem. Process Des. Dev. 25, 898-907. 